\title{
The electrophysiological correlates sustaining the retrieval of face-name associations: An ERP study
}

\author{
F. JOASSIN, ${ }^{\mathrm{a}, \mathrm{b}}$ S. CAMPANELLA,,${ }^{\mathrm{a}, \mathrm{b}}$ D. DEBATISSE, ${ }^{\mathrm{b}}$ J.M. GUERIT, ${ }^{\mathrm{c}}$ R. BRUYER, ${ }^{\mathrm{a}}$ AND \\ M. CROMMELINCK ${ }^{\mathrm{b}}$ \\ ${ }^{a}$ Unité de Neurosciences Cognitives (NESC), Faculté de Psychologie et des Sciences de l'Education, Université catholique de \\ Louvain, Louvain-la-Neuve, Belgium \\ ${ }^{b}$ Laboratoire de Neurophysiologie (NEFY), Faculté de Médecine, Université catholique de Louvain, Brussels, Belgium \\ ${ }^{c}$ Unité de Neurophysiologie Clinique, Cliniques Universitaires St-Luc et Unité de Neurochimie (NCHM), Faculté de Médecine, \\ Université catholique de Louvain, Brussels, Belgium
}

\begin{abstract}
An ERP study on 9 healthy participants was carried out to temporally constrain the neural network proposed by Campanella et al. (2001) in a PET study investigating the cerebral areas involved in the retrieval of face-name associations. Three learning sessions served to familiarize the participants with 24 face-name associations grouped in 12 male/female couples. During EEG recording, participants were confronted with four experimental conditions, requiring the retrieval of previously learned couples on the basis of the presentation of name-name $(\mathrm{NN})$, face-face $(\mathrm{FF})$, name-face $(\mathrm{NF})$, or face-name $(\mathrm{FN})$ pairs of stimuli. The main analysis of this experiment consisted in the subtraction of the nonmixed conditions ( $\mathrm{NN}$ and FF) from the mixed conditions (NF and FN). It revealed two main ERP components: a negative wave peaking at left parieto-occipital sites around $285 \mathrm{~ms}$ and its positive counterpart recorded at left centro-frontal electrodes around $300 \mathrm{~ms}$. Moreover, a dipole modeling using three dipoles whose localization corresponded to the three cerebral areas observed in the PET study (left inferior frontal gyrus, left medial frontal gyrus, left inferior parietal lobe) explained more than $90 \%$ of the variance of the results. The complementarity between anatomical and neurophysiological techniques allowed us to discuss the temporal course of these cerebral activities and to propose an interactive and original anatomo-temporal model of the retrieval of face-name associations.
\end{abstract}

Descriptors: Event-related potentials, Faces, Names, Associative processes, Dipole localization

The ability to recognize familiar people and to recall specific information about them, such as their names, plays a fundamental role in all our social interactions. Because these processes are highly reliable and automatic, one could imagine that they are quite simple (Sergent, Ohta, \& MacDonald, 1992). However, several recent studies have shown, for instance, that the ability to recognize a person and to recall her or his name is sustained by distinct brain areas. As distinct neural regions appear to be engaged in these different recognition processes, how the outputs of these diverse processes are integrated in a single everyday life experience is still matter of debate (Damasio, 1989).

Face processing is a well-documented research area. Studies of brain-damaged patients have shown that prosopagnosia, a specific impairment of face recognition (Bodamer, 1947), is often

This study was supported by FRSM No. 3.4550.02. The first and second authors are supported by the Belgian Fund of Scientific Research (FNRS).

Address reprint requests to: F. Joassin, Ph.D. student, Unité de Neurosciences Cognitives (PSP/NESC), Place Cardinal Mercier, 10, B 1348 Louvain-la-Neuve, Belgium. E-mail: frederic.joassin@psp.ucl.ac.be. associated with lesions of occipito-temporal regions, and in particular with lesions of right lingual and fusiform gyri (De Renzi, Peroni, Calesimo, Silveri, \& Fazio, 1994; Takahashi, Kawamura, Hirayama, Shiota, \& Tsono, 1995). PET and fMRI activation studies investigating the perception of faces support these results. They generally show an enhanced activation of bilateral extrastriate regions, including the inferior temporal gyrus (Puce, Allison, Gore, \& McCarthy, 1995) and the lingual and fusiform gyri (Dubois et al., 1999; Haxby et al., 1994; Puce, Allison, Asgari, Gore, \& McCarthy, 1996; Sergent et al., 1992), with a predominance of the right hemisphere (Kanwisher, McDermott, \& Chun, 1997; Kim et al., 1999).

The processes involved in proper name retrieval are less clearly circumscribed. Studies of patients suffering from proper name anomia, an inability to recall and produce people's name while semantic and biographical information about people remains accessible (Semenza \& Zettin, 1988), have revealed lesions of the left medial and anterior temporal lobe (Damasio, Damasio, Tranel, \& Brandt, 1990; Papagno \& Capitani, 1998; Reinkemeier, Markowitsch, Rauch, \& Kessler, 1997). Brain imaging studies of healthy subjects also support these results, as 
people's names retrieval is associated with a greater activation of left middle temporal gyrus (Damasio, Grabowski, Tranel, Hichwa, \& Damasio, 1996), and more specifically with activation of its anterior portion, corresponding to Brodmann area (BA) 21 (Tempini et al., 1998).

Overall, there is clear evidence that distinct cortical regions mediate face and proper name processing. These observations lead us to ask the question of how the brain operates to integrate these distinct face and name representations to form a unified representation of familiar people. First, one can wonder how people are able to encode and store such an integrated face-name representation in long-term memory. Several studies seem to outline the key role of the hippocampus as mediating the relational bound created between face and name representations (Cohen et al., 1999; Leveroni et al., 2000; Sperling et al., 2001). Second, one can also wonder how people are able to retrieve such face-name associations. This question has recently been examined by Campanella et al. (2001), by means of a PET study. Participants had to learn 24 unknown faces associated with 24 family names and to group the 24 so-created people in 12 male/ female couples (e.g., male CORNET/female BODART). In this way, subjects were able to retrieve the representation of a face (face CORNET) when presented with the name of the associated person (name BODART), and conversely. During PET recording, the task was to decide whether each presented pair of stimuli was a previously learned couple or not. Results showed that recognition of face-name associations, relative to recognition of face-face and name-name associations, activated a neural network lateralized in the left hemisphere and included the inferior frontal gyrus (BA 45), the medial frontal gyrus (BA 6), and the inferior parietal lobe (BA 40). In accordance with other studies (Clark et al., 2000), the authors suggested that the activation of left BA40 could be interpreted as the locus where the association of the distinct visual representations presented to subjects took place.

Nevertheless, the PET technique, due to its poor temporal resolution, does not allow us to infer any information about the timing of occurrence of these activations. As a major aim of neurocognitive studies is to delineate when the different processes of a cognitive function take place in the brain, it was therefore relevant to replicate the study of Campanella et al. (2001) by using event-related potentials (ERPs; Ruggs \& Coles, 1995). ERPs are defined as an imaging technique characterized by a high temporal resolution, which would allow us to neurophysiologically index the cerebral processes involved in the retrieval of a face-name association.

Indeed, if the psychophysiology of face perception and proper name processing have already been studied, we know of no study that has examined the temporal course of the retrieval of facename associations. On the one hand, face perception is classically referred to two early electrophysiological components, known as the P1/N1 and the N170/VPP. The first complex is composed by a positive peak (P1) culminating around $100 \mathrm{~ms}$ at posterior electrodes $(\mathrm{Oz})$ and a centro-frontal $(\mathrm{Cz})$ negativity $(\mathrm{N} 1)$ occurring with a similar latency. The $\mathrm{P} 1 / \mathrm{N} 1$ complex is typically described in visual ERP studies to reflect early striate and extrastriate visual processing (Campanella, Quinet, Bruyer, Crommelinck, \& Guérit, 2002; Gomez, Clark, Luck, Fan, \& Hillyard, 1994; Heinze et al., 1994). The N170/VPP complex is composed of a negativity peaking bilaterally around $150 \mathrm{~ms}$ (N170) at temporal sites (T5 and T6; Bentin, Allison, Puce, Perez, \& McCarthy, 1996; Campanella et al., 2000; George,
Evans, Fiori, Davidoff, \& Renault, 1996). This negativity reverses polarity at the level of $\mathrm{Cz}$ and gives rise to the VPP (Bötzel, Schulze, \& Stodieck, 1995; Jeffreys, 1989; Rossion et al., 1999). The N170/VPP is considered as the process indexing the structural analysis of facial information to obtain a configurational face representation (Jeffreys, 1996).

On the other hand, ERP studies concerning proper name processing are less numerous than for faces. Nevertheless, it seems that the perception of proper names elicited a bilateral P1 on temporo-occipital sites, a left-lateralized N1, and a large P2 at vertex (Dehaene, 1995; Müller \& Kutas, 1996; Proverbio, Lilli, Semenza, \& Zani, 2001). Moreover, Dehaene observed ERP differences beginning to appear around $260 \mathrm{~ms}$ after stimulus onset, with a larger left inferior temporal negativity for proper names relative to other types of written words (animal names, numerals, and verbs).

With all these considerations in mind, the main issue of this research was to assess the existence of specific electrophysiological waves associated with the retrieval of face-name associations, in order to temporally constrain the neuroanatomical model proposed by Campanella et al. (2001). In the present experiment, we used the same methodology as in the PET study. Participants were first submitted to a learning phase. Then, during EEG recording, they were confronted with four experimental conditions: (1) a "name-name" condition (NN), in which they were visually presented with pairs composed of a name followed by another name; (2) a "face-face" condition (FF); (3) a "name-face" condition (NF); and (4) a "face-name" condition $(\mathrm{FN})$, in which they were presented with pairs composed of a name and a face. Their task always consisted of deciding whether each presented couple corresponded to one of the previously learned male/female couples. It is worth noting that all displayed stimuli were familiar. So, when a new couple was presented, it was nevertheless formed with two names, two faces, or a name and a face previously learned but issuing from two different couples. Also, the pairs were always constituted by a male and a female stimulus.

Using the same material and task across all conditions ensures an identical balance over task conditions, which allowed us to make three different assumptions. First, we should observe the early electrophysiological peaks classically described in the literature and associated with the perceptual processing of faces (P1/N1 and N170/VPP) and proper names (P1/N1, P2). Moreover, if, as expected, conditions shared the same level of difficulty and required identical attentional ressources, we should not observe any significant latency or amplitude differences between waves associated with the first and second stimuli of each pair (first names of NN and NF conditions, first faces of FF and FN conditions, second names of $\mathrm{NN}$ and $\mathrm{FN}$ conditions, second faces of FF and NF conditions) Second, we use a paradigm in which two experimental conditions were defined by two stimuli presenting the same visual format ( $\mathrm{NN}$ and FF), whereas two other conditions were defined by two stimuli presenting two different visual formats ( $\mathrm{NF}$ and $\mathrm{FN}$ ). In other words, subjects had to make a judgment (correct male/female couple or not) when confronted with the second stimuli of these pairs, and this second stimuli shared (FF and NN) or did not share (FN and NF) the visual format of the first one. It can therefore be hypothesized that the subtraction of the second stimuli of NN and FF conditions from the second stimuli of NF and FN conditions would reveal the neurophysiological mechanisms specifically implicated in the retrieval of an association 
composed by distinct face and name visual representations. From the results of the PET study of Campanella et al. (2001), we can further assume that these neurophysiological activities (1) will be generated in the left hemisphere, and (2) will reflect specific integrative processes (of visual facial representation with visual name representation), distinct from those implied in the processing of either a face or a name. It is worth noting that both NN and FF conditions could lead to implicit activations of the cerebral regions sustaining these specific integrative processes, but it has been shown that the explicit processing of information gives rise to enhanced cerebral activations relative to the implicit processing of this information (Price, Moore, Humphreys, Frackowiak, \& Friston, 1996; Price, Moore, Humphreys, \& Wise, 1997). Moreover, this subtraction method has already received strong empirical support by using ERPs (e.g., Dehaene et al., 2001; Giard \& Peronnet, 1999). Third, even if the spatial resolution of the ERP technique is poorer than the one of PET or fMRI, a dipole localization analysis of the specific integrative waves revealed by the subtraction could (1) give prominence to neural generators spatially close from the three activated neural regions found by Campanella et al. (2001), and (2) reveal a precise timing of their order of occurrence.

\section{Methods}

\section{Participants}

Nine paid volunteers (5 men), who did not take part in the previous PET study, participated in the present experiment. They were between 20 and 29 years old (mean: 22.8 years) and were all right-handed but one. They all had a normal or corrected-tonormal vision and no one had a previous or present history of significant medical illness. All participants gave their written informed consent.

\section{Stimuli}

We used the same stimuli as in the PET study, that is, 24 unknown faces (12 females) selected from the "Psychological Image Collection at Stirling (PICS)" at the website address http://www.pics.psych.stir.ac.uk. All faces were presented in frontal position with a neutral expression. They were downloaded into a Macintosh computer and were edited by Adobe Photoshop 5.0 (Adobe Systems Incorporated). Gray-scale images were created and scaled to $274 \times 350$ pixels $(123 \times$ $96 \mathrm{~mm}$, corresponding to a visual angle of $7.04^{\circ} \times 5.5^{\circ}$ ). A set of 24 proper names (those used in our previous study) were chosen from the "Belgian National Institute of Statistics" so that each name had nearly the same frequency of occurrence in the Belgian population and was made up of six letters and two syllabes. Gray-scale images of $274 \times 350$ pixels $(123 \times 96 \mathrm{~mm}$, visual angle of $7.04^{\circ} \times 5.5^{\circ}$ ) were also created with Adobe Photoshop 5.0 for these names. Then the 24 faces were randomly paired with the 24 names and each male "face-name association" was coupled with a female one (Figure 1).

\section{Procedure}

Participants were presented with the same learning phase, defined by three sessions in the three consecutive days preceding the EEG recording session. Each learning session lasted about $1 \mathrm{~h}$. The first day, they were shown 12 faces ( 6 females) associated with their respective names on a monitor. Participants were asked to encode these associations, with no time pressure. Then,

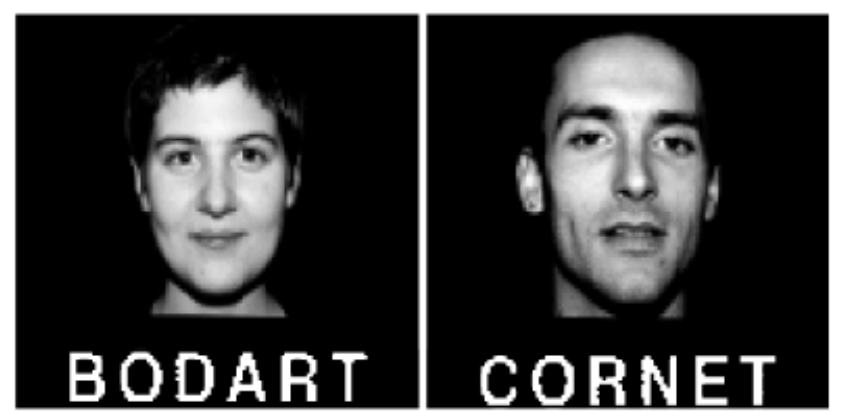

Figure 1. Illustration of a couple (female/male) face-name association.

they were given sheets of paper with the names and faces just encoded and they had to re-create the face-name pairs as accurately and quickly as possible. If they performed the task correctly, the 12 learned face-name associations were then presented in six different male/female couples, in order to encode these associations. Following that, sheets of paper with the names and faces encoded were again given to participants who had to form the six couples as accurately and quickly as possible. The session ended when they were able to complete this last task with no error or hesitation at all (if they were not, a new encoding phase was performed and the task was again proposed). The second learning session was dedicated to the encoding of the other 12 face-names associations and the six resulting couples. The same procedure as in the first session was applied. On the third day, participants were presented with the 24 face-name associations and the $12 \mathrm{male} /$ female couples. This recapitulation session used the same tasks as previously and was ended when participants were able to retrieve all the couples quickly and totally accurately.

On the fourth day, EEG was recorded in four different experimental conditions. These were matching tasks after namename $(\mathrm{NN})$, face-face $(\mathrm{FF})$, face-name $(\mathrm{FN})$, and name-face (NF) presentations. The stimuli were presented as male-female couples. A trial was defined by a small white cross during $300 \mathrm{~ms}$, the first stimulus presented for $700 \mathrm{~ms}$, a black screen as interstimulus for $500 \mathrm{~ms}$, the second stimulus for $700 \mathrm{~ms}$, and a final black screen for $1,000 \mathrm{~ms}$. In the nonmixed conditions, the two stimuli were two names (NN) or two faces (FF), whereas in the mixed conditions, participants were confronted with either a face followed by a name (FN) or by a name followed by a face (NF). Participants had to decide whether the pairs of stimuli referred correctly to a previously learned couple (e.g., if the name Bodart was followed by the face Cornet, the correct response is "yes"; however, a "no" response would have been required if, e.g., the name "Bodart" had been presented with the male face "Jansen"). To do so, they had to press one of two predefined keys (counterbalanced across participants) on a response button box with the right finger. They were confronted with 576 trials (144 per condition), randomly presented in 24 blocks. Half of the trials were incorrect. None of the incorrect couples appeared more than once and an incorrect couple was never defined by two stimuli representing the same person or the same sex (e.g., two males). During EEG recording, participants were sitting on a chair in a dark and quiet room with their heads restrained by a chin rest. Their heads were placed $100 \mathrm{~cm}$ from a high-resolution computer screen where stimuli were presented. Written instructions were given to participants, with emphasis on speed and 
accuracy. Before the first block, some practice trials were given to participants to refamiliarize them with the task.

In contrast to the PET study, where conditions were presented by block, here we chose to mix the four conditions in the same proportions within each blockr to maintain identical levels of attention and prevent some anticipatory effects across trials.

\section{EEG Recordings}

Sixty-four electrodes mounted in an electrode cap (Quick-Cap, NeuroMedical Supplies, Neurosoft, Inc.) recorded EEG. Electrode positions included the standard 10-20 system locations and additional intermediate positions. Recordings were made with the two ears as the physical reference. The EEG was amplified by battery-operated SYNAMPS amplifiers with a gain of 30,000 and a band pass of 0.015 to $30 \mathrm{~Hz}$. EEG was continuously recorded (sampling rate $500 \mathrm{~Hz}$; Neuroscan) and stored on disk for further analyses. Trials with EOG artifacts were automatically eliminated and epochs beginning $150 \mathrm{~ms}$ prior to stimulus onset and continuing for $850 \mathrm{~ms}$ were created. A recalculation was made to obtain common average reference recordings (Bertrand, Perrin, \& Perrier, 1985). Codes synchronized with stimulus delivery were used to average selectively epochs associated with different stimulus types. Three parameters were coded for every stimulus: (1) the condition (FF, NN, FN, or NF), (2) the position of the stimulus in the pair (first or second), and (3) the kind of pair (correct or incorrect pair). This coding allowed us to compute different averages of ERP target stimuli. These averages were made for each participant individually. A sample grand average was obtained by averaging across the participants first names of $\mathrm{NN}$, first names of $\mathrm{NF}$, second names of $\mathrm{NN}$, second names of FN, first faces of FF, first faces of FN, second faces of FF, and second faces of NF conditions, second stimuli of $\mathrm{NN}$ and FF conditions, and second stimuli of FN and NF conditions. Only correct trials were included in these averages.

\section{Statistical Analyses}

Latencies and percentages of correct responses were computed and analyzed with Systat 5.2.1 (Systat, Inc.). To ensure that the mixed ( $\mathrm{NF}$ and $\mathrm{FN}$ ) and the nonmixed (FF and $\mathrm{NN}$ ) conditions shared identical levels of difficulty and attention, we compared electrophysiological waves elicited by the visual processing of the first (and second) faces of FF and FN (NF) conditions and the first (and second) names of NN and NF (NF) conditions. At selected electrodes, individual peak amplitudes of different components were obtained individually. These values were tested using paired Student $t$ tests and repeated-measures ANOVAs.

We also compared the first names and faces of mixed and nonmixed conditions at later latencies to check if one kind of condition led to preparatory and anticipative mechanisms relative to another that could explain some of the results of the subtraction. For each participant, mean amplitudes were computed at $\mathrm{Oz}, \mathrm{Pz}, \mathrm{Cz}$, and $\mathrm{Fz}$ within four time windows (300$398 \mathrm{~ms}, 400-498 \mathrm{~ms}, 500-598 \mathrm{~ms}, 600-698 \mathrm{~ms}$, one measure every $2 \mathrm{~ms}$ ). Repeated-measures ANOVAs were then calculated between names of NN and NF conditions and faces of FF and NF conditions for each time interval.

The major analysis of this experiment was the subtraction of the second stimuli of NN and FF (nonmixed) conditions from the second stimuli of FN and NF (mixed) conditions to assess specific integrative processes. Mixed and nonmixed conditions were averaged and subtracted in each subject, and peak amplitudes and latencies were measured for each electrode of interest. Significant effects were then calculated on the grand average at each selected electrode using Student $t$ tests (amplitude of the difference wave compared to zero). The spatio-temporal patterns that had a significant amplitude $(p<.01)$ at at least one electrode for $15 \mathrm{~ms}$ (Rugg, Doyle, \& Wells, 1995) were considered as significant.

The analysis of intracranial dipoles was performed with the ASA software (A.N.T. Software BV, Entschende, The Netherlands), which determines the position and orientation of intracranial dipoles and their time-varying strength by using a three-layer spherical head model. We used a rotating dipole method in which the position of the dipoles was fixed but the orientation remained free.

\section{Results}

\section{Behavioral Data}

An ANOVA with kind of conditions (mixed conditions: mean of $\mathrm{NF}$ and FN, nonmixed conditions: mean of NN and FF) and kind of pairs (correct, i.e., learned vs. incorrect) as within factors was performed on percentages of correct responses (Table 1). There were significant effects of the kind of condition, $F(1,8)=23.008, p=.001$, the kind of pair, $F(1,8)=10.028$, $p=.034$, and interaction between kind of condition and kind of pair, $F(1,8)=10.028, p=.034$. The main effect of the kind of condition indicates that the mixed conditions were better performed than the nonmixed conditions. But, as shown in Table 1, all conditions were highly performed (above 94\%) and no difference between conditions exceeded 3.1\%. Moreover, the segregation of the four conditions reveals that all but FF conditions were identically performed (correct NN: $97 \%$, incorrect NN: 97\%; correct FF: $96 \%$, incorrect FF: $92 \%$; correct NF: 97\%, incorrect NF: 99\%; correct $\mathrm{FN}$ : $98 \%$, incorrect FN: $96 \%$ ) The main effect of response type show that subjects performed the task more efficiently when the presented pairs corresponded to a previously learned couple. However, paired Student $t$ tests showed that this difference was only significant for the nonmixed conditions, $T(8)=2.53, p=.04$, which explains the significant interaction.

The same ANOVA was performed on correct response latencies (Table 1). There was a main effect of the kind of pair, $F(1,8)=13.43, p=.01$, but no significant main effect of the kind of condition, $F(1,8)=0.06$, n.s., and no significant interaction, $F(1,8)=0.71$, n.s. Thus, subjects answered faster to the previously learned pairs in mixed and nonmixed conditions.

Overall, behavioral measures showed that subjects answered better and faster for the previously learned pairs, but there was no clear evidence of major differences of complexity level or attentional ressources between mixed and nonmixed conditions.

Table 1. Mean Correct Responses and Latencies (SD) for the Correct and Incorrect Pairs of Mixed ( FN and NF) and Nonmixed ( NN and FF) Conditions

\begin{tabular}{lccccc}
\hline \hline & \multicolumn{2}{c}{ Mixed } & & \multicolumn{2}{c}{ Nonmixed } \\
\cline { 2 - 3 } \cline { 5 - 6 } Response type & Correct & Incorrect & & Correct & Incorrect \\
\hline Mean correct responses (\%) & 97.4 & 97.4 & & 96.4 & 94.3 \\
& $(1.42)$ & $(1.13)$ & & $(1.88)$ & $(1.73)$ \\
Mean correct latencies (ms) & 522 & 577 & & 526 & 569 \\
& $(154.9)$ & $(155.9)$ & & $(137.4)$ & $(146.9)$ \\
\hline \hline
\end{tabular}




\section{Electrophysiological Data}

Visual processing of faces and names

The purpose of this first set of analyses was to ensure that all faces and names were encoded in the same way across mixed and nonmixed conditions.

Faces. For faces, we considered the P1/N1 and the N170/ VPP complexes (Figure 2a, left part), reflecting the structural encoding processes as described in the literature. The P1/N1 complex was characterized for all faces by a positivity over posterior electrodes culminating $(\mathrm{Oz})$ at $118 \mathrm{~ms}$ and a polarity reversal at central sites $(\mathrm{Cz})$ at the same latency. The N170 wave was observable bilaterally at parieto-occipital sites. It culminated at PO7 and PO8 at $161 \mathrm{~ms}$ and was synchronized with a Vertex Positive Potential maximally recorded at $\mathrm{Cz}$ at $162 \mathrm{~ms}$.

At first, ERPs elicited by the first faces of the FF condition were compared to ERPs elicited by the first faces of the FN condition. An ANOVA on peak voltage amplitude of P1 with conditions $(\mathrm{FF}, \mathrm{FN})$ and lateralization $(\mathrm{O} 1, \mathrm{O} 2)$ as factors was carried out. We report here only the significant effects. The complete results of the ANOVAs are summarized in Table 2. It showed a significant effect of lateralization, $F(1,8)=7.15, p=.03$, and a significant interaction, $F(1,8)=5.78, p=.04$. A paired Student $t$ test showed that the amplitude of P1 was larger in the right hemisphere $(\mathrm{O} 2)$ only in the FF condition, $T(8)=3.71$, $p=.01$. The same analysis was performed on the negative counterpart (N1) at central electrodes. An ANOVA with conditions $(\mathrm{FF}, \mathrm{FN})$ and lateralization $(\mathrm{C} 1, \mathrm{C} 2)$ revealed a significant effect of lateralization, $F(1,8)=11.41, p=.01$. The interaction between conditions and lateralization did not reach a significant statistical level, $F(1,8)=0.32$, n.s. The amplitude of N1 was therefore larger in the left hemisphere $(\mathrm{Cl})$ in both conditions.

An ANOVA was performed on peak voltage amplitude of N170 with conditions (FF, FN) and lateralization (PO7, PO8). Factors failed to show any significant effect of condition, lateralization, or interaction. A similar analysis was applied on peak voltage amplitude of VPP. An ANOVA with condition (FF, $\mathrm{FN})$ and lateralization $(\mathrm{C} 1, \mathrm{C} 2)$ showed no significant effects.

Then, we compared the ERPs elicited by the second faces of FF and NF conditions (Figure 2a, right part). An ANOVA on peak voltage amplitude of P1 with conditions (FF, NF) and lateralization (PO7, PO8) showed a significant effect of lateralization, $F(1,8)=6.19, p=.04$, without significant effect of condition, $F(1,8)=0.28$, n.s., or interaction, $F(1,8)=0.08$, n.s., indicating that $\mathrm{P} 1$ was larger in the right hemisphere in both conditions. The ANOVAs performed on N1, N170, and VPP failed to show any significant effect.

Names. We observed the same electrophysiological waves for names as for faces, that is, a P1/N1 and a N170/P2 complex (Figure $2 \mathrm{~b}$ ) corresponding to the encoding processes of orthographical stimuli (Bentin, Mouchetant-Rostaing, Giard, Echallier, \& Pernier, 1999). The $\mathrm{P} 1$ positivity peaked at the $\mathrm{Oz}$ electrode between 101 and $105 \mathrm{~ms}$ and the reversal polarity was maximum at $\mathrm{Cz}$ between 107 and $109 \mathrm{~ms}$. The N170 wave appeared bilaterally at parieto-occipital electrodes (PO7, PO8) at $155 \mathrm{~ms}$ and was synchronized with a P2 wave culminating at central electrodes $(\mathrm{Cz})$ at $158 \mathrm{~ms}$.

As for faces, we compared first the ERPs elicited by the first names of NN and NF conditions (Table 2). ANOVAs on peak voltage amplitudes of $\mathrm{P} 1, \mathrm{~N} 1, \mathrm{~N} 170$, and $\mathrm{P} 2$ with conditions $(\mathrm{NN}, \mathrm{NF})$ and lateralization $(\mathrm{P} 1: \mathrm{O} 1, \mathrm{O} 2 ; \mathrm{N} 1: \mathrm{C} 1, \mathrm{C} 2 ; \mathrm{N} 170$ :
PO7, PO8; P2: C1, C2) as factors did not reveal any significant effect of condition, lateralization, or interaction. The comparison of the second names of NN and FN conditions failed also to show any significant differences between conditions or any effect of lateralization.

To summarize, the comparison of the electrophysiological waves associated with the processing of the first stimuli (faces or names) of mixed and nonmixed conditions did not reveal any significant differences between conditions, except some lateralization effects of $\mathrm{P} 1 / \mathrm{N} 1$ in conditions requiring the perception of faces. Nevertheless, the mean amplitudes of the P1/N1 and the N170/VPP (or P2 for names) complex were identical for the first faces of FF and FN conditions and for the first names of NN and NF conditions. Therefore, it seems that names and faces were processed in the same way in all conditions.

Comparison of the first stimuli of mixed and nonmixed conditions between 300 and $700 \mathrm{~ms}$

We compared mean amplitudes recorded at $\mathrm{Oz}, \mathrm{Pz}, \mathrm{Cz}$, and $\mathrm{Fz}$ in four 100-ms time windows (one measure every $2 \mathrm{~ms}$ ) after the onset of the first names of NN and NF conditions and the first faces of FF and FN conditions to ensure that one kind of condition did not create anticipatory mechanisms relative to another. Repeated-measures ANOVAs with conditions (names: $\mathrm{NN}$ and NF; faces: FF and FN) and electrode position $(\mathrm{Oz}, \mathrm{Pz}$, $\mathrm{Cz}, \mathrm{Fz})$ as factors were performed for each time window. They failed to find any significant effect at any time for the names or for the faces (Table 3).

\section{Subtraction of $N N$ and FF from $N F$ and $F N$}

This subtraction revealed two major electrophysiological components: a bilateral negativity spreading over all posterior electrodes around $284 \mathrm{~ms}$ in the left hemisphere and $293 \mathrm{~ms}$ in the right hemisphere, and a left centro-frontal positivity peaking around $300 \mathrm{~ms}$ (Figure 3a,b,c). For the negativity, a significant negative amplitude was recorded at $\mathrm{Oz}$ and POz; O1, PO3, PO5, PO7, P3, and P5 in the left hemisphere, PO4, PO6, PO8, P4, and $\mathrm{P} 2$ in the right hemisphere (for amplitudes, latencies, and significance levels, see Table 4). The centro-frontal positivity was maximally observed at $\mathrm{C} 1$ and $\mathrm{FC} 1$ and, to a lesser extent, at $\mathrm{Cz}$ and $\mathrm{FCz}$. At the corresponding electrodes of the right hemisphere ( $\mathrm{C} 2$ and $\mathrm{FC} 2$ ), the positive amplitude did not reach a significant statistical level (Table 5).

An ANOVA with lateralization (left or right hemisphere) and electrode position (occipital: O1, O2; occipito-parietal: PO5, PO6; parietal: P5, P6) as factors was performed on mean amplitudes and latencies of the negativity. It failed to find any significant difference of amplitude between hemispheres (lateralization: $F(1,8)=2.67$, n.s.) nor any effect of electrode position, $F(2,16)=1.35$, n.s., nor any interaction, $F(2,16)=3.27$, n.s. On latencies, the analysis revealed that the negativity appeared first in the left hemisphere (hemisphere: $F(1,8)=8.41, p=.02$ ) but did not reveal any significant effect of electrode position, $F(2,16)=0.31$, n.s., or interaction, $F(2,16)=0.38$, n.s. As the centro-frontal positivity was only significant in the left hemisphere, no amplitude or latency analyses of lateralization were performed on this component.

\section{Dipole modeling}

Dipole localization was carried out on the grand average file of the subtraction described above. Our goal was to temporally constrain the anatomical network whose activation was observed 
(a)
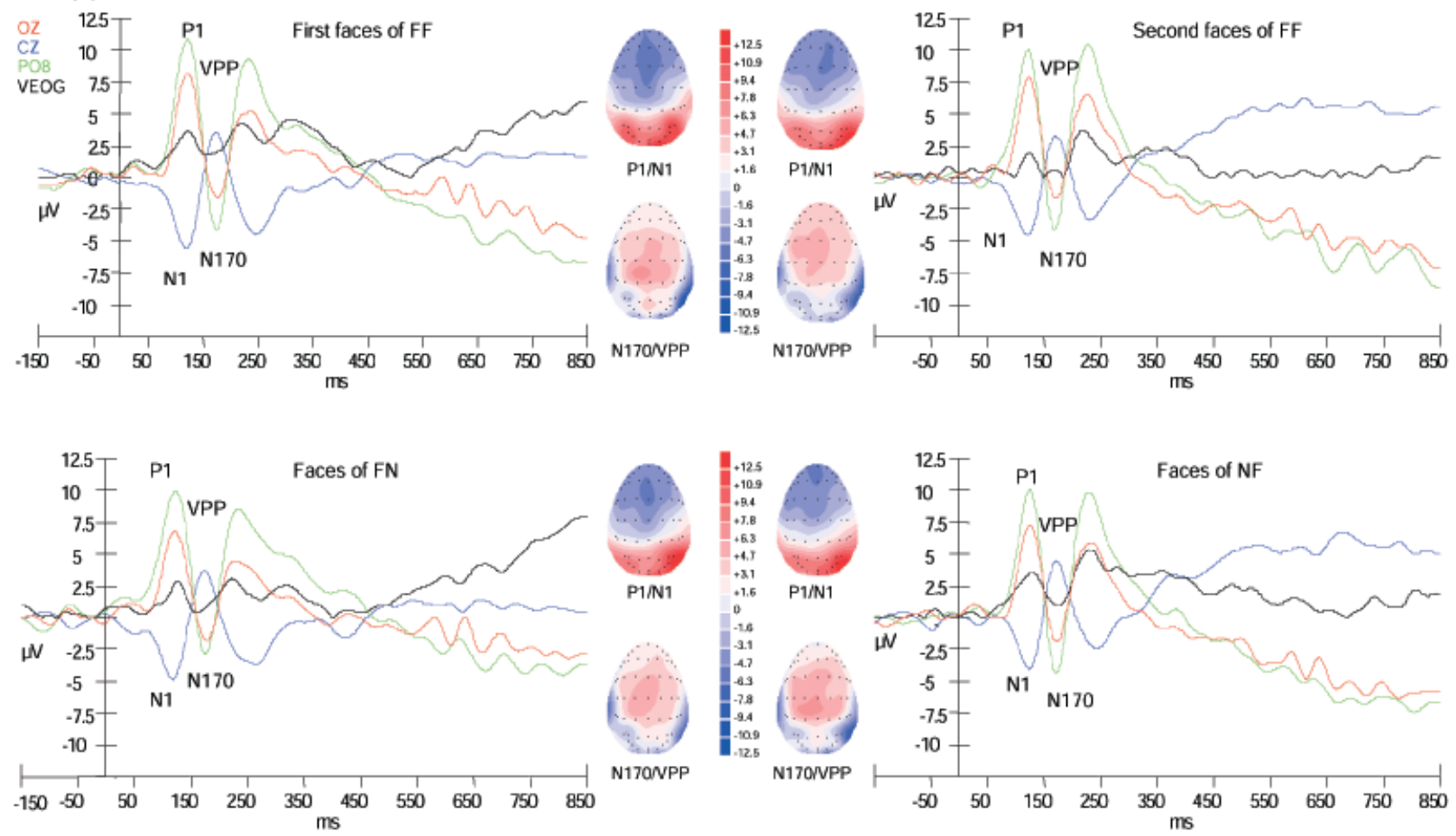

(b)
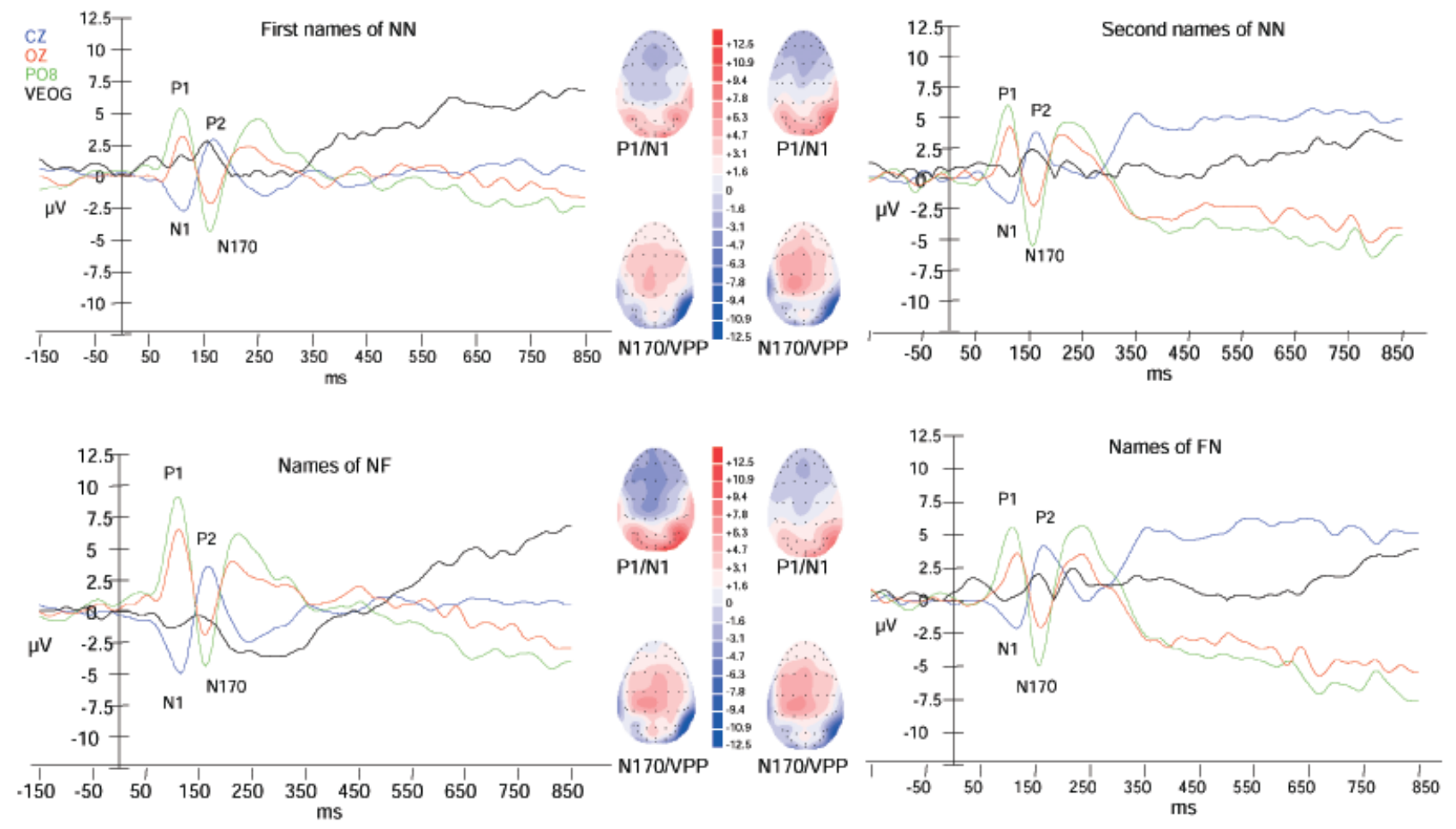

Figure 2. Illustration of grand-average ERPs of P1/N1 and N170/P2-VPP complexes elicited by (a) first and second faces of FF, FN, and NF conditions, and (b) first and second names of NN, NF, and FN conditions. For each figure, the upper topographies correspond to P1/N1 complexes and the lower to N170/P2 (or VPP) complexes.

by Campanella et al. (2001). In a simultaneous fit of all source analyses, we constrained the model with three rotating dipoles whose localization corresponded to the three cerebral areas observed in the previous PET study. In the chosen time window
(260-320 ms), the three dipoles explained $90.26 \%$ of the variance (residual variance (RV): 9.74\%, correlation: 95\%; Figure 4). We then performed separate fit analyses for every 1-ms interval between 260 and $320 \mathrm{~ms}$ for each of the three dipoles to determine 
Table 2. Results of the ANOVAs Performed on Peak Voltage Amplitudes of P1, N1, N170, and VPP (or P2) with Conditions (First Faces: FF, FN; Second Faces: FF, NF; First Names: NN, NF; Second Names: NN, FN) and Lateralization (P1: O1, O2; N1: C1; C2; N170: PO7, PO8; VPP-P2: C1, C2) as Factors

\begin{tabular}{|c|c|c|c|c|c|c|c|}
\hline & & \multicolumn{3}{|c|}{ First faces $(\mathrm{FF}-\mathrm{FN})$} & \multicolumn{3}{|c|}{ Second faces (FF-NF) } \\
\hline & & Condition & Lateralization & Interaction & Condition & Lateralization & Interaction \\
\hline \multirow[t]{9}{*}{ a. } & $\mathrm{P} 1$ & $F(1,8)=2.86$ & $F(1,8)=7.15$ & $F(1,8)=3.71$ & $F(1,8)=0.28$ & $F(1,8)=6.19$ & $F(1,8)=0.08$ \\
\hline & N1 & $\begin{array}{c}\text { n.s. } \\
F(1,8)=0.1\end{array}$ & $\begin{array}{c}\boldsymbol{p}=\mathbf{. 0 3} \\
F(1,8)=11.41\end{array}$ & $\begin{array}{c}\boldsymbol{p}=. \mathbf{0 1} \\
F(1,8)=0.32\end{array}$ & $\begin{array}{c}\text { n.s. } \\
F(1,8)=0.25\end{array}$ & $\begin{array}{c}\boldsymbol{p}=. \mathbf{0 4} \\
F(1,8)=0.89\end{array}$ & $\begin{array}{c}\text { n.s. } \\
F(1,8)=0.06\end{array}$ \\
\hline & & & $p=.01$ & & & & \\
\hline & N170 & $F(1,8)=2.82$ & $F(1,8)=0.59$ & $F(1,8)=1.14$ & $F(1,8)=1.63$ & $F(1,8)=1.80$ & $F(1,8)=1.21$ \\
\hline & & & & & $\begin{array}{c}\text { n.s. } \\
\text { (n) }\end{array}$ & & \\
\hline & VPP & $F(1,8)=0.27$ & $F(1,8)=0.28$ & $F(1,8)=1.3$ & $F(1,8)=0.03$ & $F(1,8)=2.18$ & $F(1,8)=2.34$ \\
\hline & & n.s. & n.s. & n.s. & n.s. & n.s. & n.s. \\
\hline & & \multicolumn{3}{|c|}{ First names (NN-NF) } & \multicolumn{3}{|c|}{ Second names $(\mathrm{NN}-\mathrm{FN})$} \\
\hline & & Condition & Lateralization & Interaction & Condition & Lateralization & Interaction \\
\hline \multirow[t]{8}{*}{ b. } & $\mathrm{P} 1$ & $F(1,8)=1.2$ & $F(1,8)=3.04$ & $F(1,8)=4.99$ & $F(1,8)=0.07$ & $F(1,8)=3.95$ & $F(1,8)=4.16$ \\
\hline & & n.s. & n.s. & n.s. & n.s. & n.s. & n.s. \\
\hline & N1 & $F(1,8)=0.59$ & $F(1,8)=0.34$ & $F(1,8)=4.49$ & $F(1,8)=0.18$ & $F(1,8)=0$ & $F(1,8)=2.06$ \\
\hline & & n.s. & n.s. & n.s. & n.s. & n.s. & n.s. \\
\hline & N170 & $F(1,8)=3.66$ & $F(1,8)=0.06$ & $F(1,8)=0.12$ & $F(1,8)=0.86$ & $F(1,8)=0.15$ & $F(1,8)=0.63$ \\
\hline & & n.s. & n.s. & n.s. & n.s. & n.s. & n.s. \\
\hline & P2 & $F(1,8)=0$ & $F(1,8)=0.57$ & $F(1,8)=0.67$ & $F(1,8)=0.11$ & $F(1,8)=0.52$ & $F(1,8)=0.05$ \\
\hline & & n.s. & n.s. & n.s. & n.s. & n.s. & n.s. \\
\hline
\end{tabular}

$P$ values above .05 were considered nonsignificant (n.s.).

when each neural generator maximally contributed to the observed electrophysiological activity. The dipole corresponding to the left parietal lobe (BA 40; $x:-50, y: 44, z: 32$ ) showed a lower RV than the other two for all intervals with a minimum RV at $298 \mathrm{~ms}$ (RV: $14.1 \%$, correlation: $92.68 \%$ ). The dipole localized in the left medial frontal gyrus (BA 6; $x: 8, y: 34, z$ : 56) showed a minimum RV at $286 \mathrm{~ms}$ (RV: $27.01 \%$, correlation: $85.43 \%$ ) and the dipole corresponding to the left inferior frontal gyrus (BA 45; $x: 36, y: 28, z: 6$ ) had a minimum RV at $310 \mathrm{~ms}$ (RV: $24.64 \%$, correlation: $86.81 \%$ ).

\section{Discussion}

The aim of the present study was to investigate the temporal course of the cerebral activity implied in the retrieval of facename associations, when subjects had to recognize couples formed by two stimuli belonging to the same visual format (two names or two faces) or not (a face and a name). We first assumed that we should observe the classical electrophysiological waves elicited by the perceptual processing of faces and names (P1/N1 and N170/VPP or P2 complexes), and that, if the four conditions

Table 3. Results of the ANOVAs Performed on the Mean Voltage Amplitudes Measured between 300 and 398 ms, 400 and $498 \mathrm{~ms}, 500$ and $598 \mathrm{~ms}$, and 600 and $698 \mathrm{~ms}$ after the Onset of the First Names and Faces of Mixed and Nonmixed Conditions

\begin{tabular}{|c|c|c|c|c|}
\hline & Milliseconds & $\begin{array}{l}\text { Electrode } \\
\text { position }\end{array}$ & Condition & Interaction \\
\hline \multirow{4}{*}{$\begin{array}{l}\text { First names } \\
\text { of NN } \\
\text { and NF }\end{array}$} & $300-398$ & $F(3,24)=0.74$ & $F(1,8)=2.52$ & $F(3,24)=0.94$ \\
\hline & $400-498$ & $\begin{array}{c}\text { n.s. } \\
F(3,24)=0.42\end{array}$ & $\begin{array}{c}\text { n.s. } \\
F(1,8)=0.18\end{array}$ & $\begin{array}{c}\text { n.s. } \\
F(3,24)=0.09\end{array}$ \\
\hline & $500-598$ & $\begin{array}{c}\text { n.s. } \\
F(3,24)=0.03\end{array}$ & $\begin{array}{c}\text { n.s. } \\
F(1,8)=0.77\end{array}$ & $\begin{array}{c}\text { n.s. } \\
F(3,24)=0.06\end{array}$ \\
\hline & $600-698$ & $\begin{array}{c}\text { n.s. } \\
F(3,24)=1.83\end{array}$ & $\begin{array}{c}\text { n.s. } \\
F(1,8)=0.94\end{array}$ & $\begin{array}{c}\text { n.s. } \\
F(3,24)=1.07\end{array}$ \\
\hline \multirow{4}{*}{$\begin{array}{l}\text { First faces } \\
\text { of FF } \\
\text { and FN }\end{array}$} & $300-398$ & $\begin{array}{c}\text { n.s. } \\
F(3,24)=0.13\end{array}$ & $\begin{array}{c}\text { n.s. } \\
F(1,8)=2.10\end{array}$ & $\begin{array}{c}\text { n.s. } \\
F(3,24)=1.80\end{array}$ \\
\hline & $400-498$ & $\begin{array}{c}\text { n.s. } \\
F(3,24)=0.88\end{array}$ & $\begin{array}{c}\text { n.s. } \\
F(1,8)=0.01\end{array}$ & $\begin{array}{c}\text { n.s. } \\
F(3,24)=1.29\end{array}$ \\
\hline & $500-598$ & $\begin{array}{c}\text { n.s. } \\
F(3,24)=035\end{array}$ & $\begin{array}{c}\text { n.s. } \\
F(1,8)=0.12\end{array}$ & $\begin{array}{c}\text { n.s. } \\
F(3,24)=0.93\end{array}$ \\
\hline & $600-698$ & $\begin{array}{c}\text { n.s. } \\
F(3,24)=2.14 \\
\text { n.s. }\end{array}$ & $\begin{array}{c}\text { n.s. } \\
F(1,8)=0.86 \\
\text { n.s. }\end{array}$ & $\begin{array}{c}\text { n.s. } \\
F(3,24)=0.63 \\
\text { n.s. }\end{array}$ \\
\hline
\end{tabular}

Factors: electrode position $(\mathrm{Oz}, \mathrm{Pz}, \mathrm{Cz}, \mathrm{Fz})$ and condition (NN and NF for the names, FF and $\mathrm{FN}$ for the faces). $P$ values above .05 were considered nonsignificant (n.s.). 
(a)
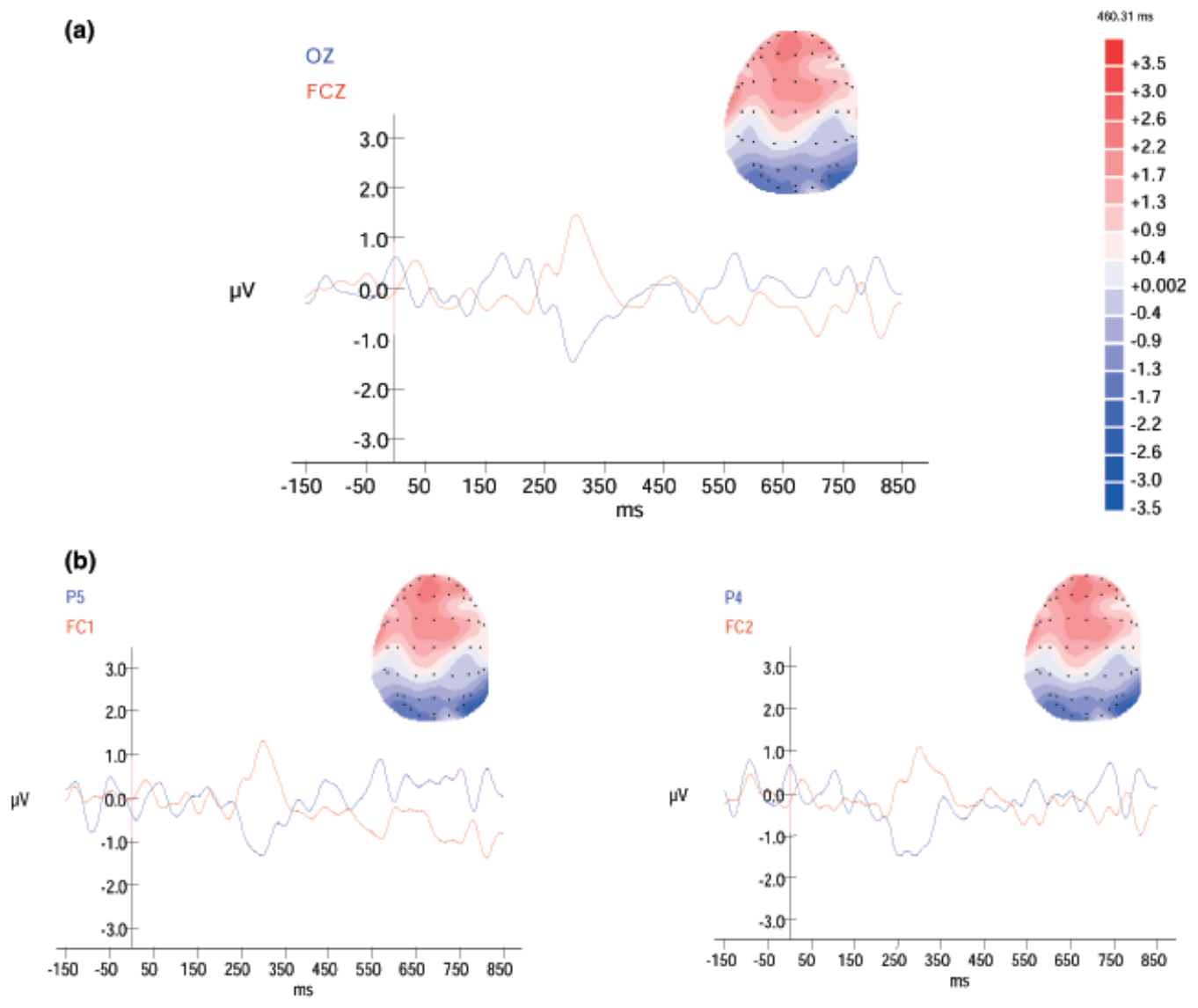

(c)

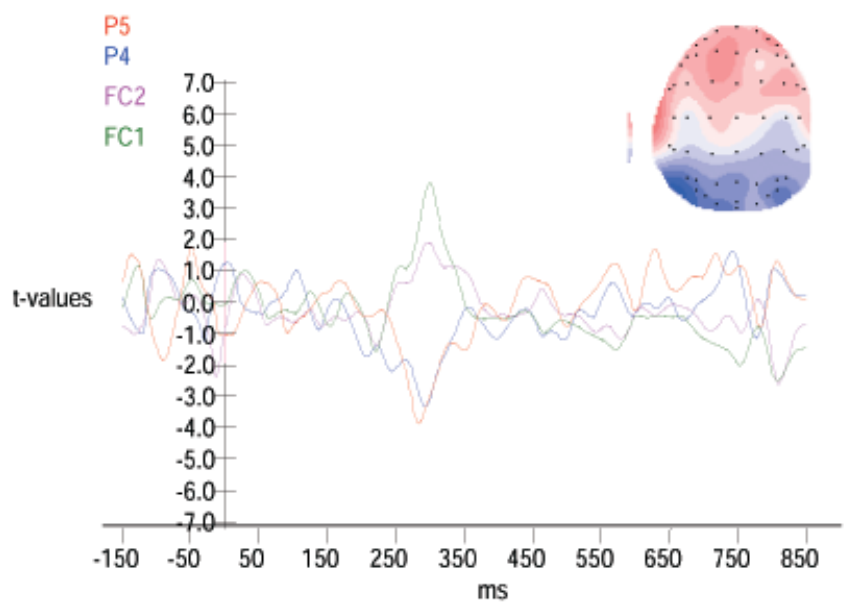

Figure 3. Illustration of grand average ERPs and topographies elicited by the subtraction of NN and FF conditions from FN and NF conditions. a: Superposition of the negative wave (in microvolts) recorded at Oz around $296 \mathrm{~ms}$ and the positive wave recorded at $\mathrm{FCz}$ around $301 \mathrm{~ms}$. b: Comparison of the ERP components (in microvolts) recorded in the left (P5 and FC1) and the right (P4 and FC2) hemispheres. c: Comparison (in $t$ values) of the positive wave recorded in the left (FC1: maximum amplitude difference [MAD] at $300 \mathrm{~ms}, p<.005)$ and right (FC2: MAD at $297 \mathrm{~ms}, p<.05)$ hemispheres and the negative wave recorded in the left (P5: MAD at $284 \mathrm{~ms}, p<.0025)$ and right $(\mathrm{P} 4:$ MAD at $294 \mathrm{~ms}, p<.005)$ hemispheres.

were matched for difficulty and attention, no amplitude or latency differences should exist between the stimuli of mixed (NF and FN) and nonmixed (NN and FF) conditions. These early ERP components are known to be sensitive to modulations of attentional processes (Coull, 1998; Itier \& Taylor, 2002). But, as suggested by statistical analyses, no difference of amplitude or latency was found between the first faces of $\mathrm{FF}$ and $\mathrm{FN}$ conditions and the first names of NN and NF conditions and between the second faces of $\mathrm{FF}$ and NF conditions and the second names of $\mathrm{NN}$ and $\mathrm{FN}$ conditions nor on the $\mathrm{P} 1 / \mathrm{N} 1$ complex nor on the N170/VPP (or P2 for names) complex. The only effect we observed was a lateralization effect on the P1/N1 complex in 
Table 4. Negativity in $(N F+F N)-(N N+F F)$; (1)

Hemispheres, (2) Electrodes, (3) Peak Amplitudes, (4) Latency of Maximum Amplitude Difference from Zero (Student t tests) and Significance Level

\begin{tabular}{llcc}
\hline \hline Electrodes & & Amplitude $(\mu \mathrm{V})$ & Latency $(\mathrm{ms})$ \\
\hline Left & O1 & -1.70 & $287^{* * *}$ \\
& PO3 & -1.79 & $284^{* * *}$ \\
& PO5 & -1.45 & $283^{* *}$ \\
& PO7 & -1.42 & $284^{* * *}$ \\
& P3 & -1.38 & $283^{* * *}$ \\
Midline & P5 & -1.35 & $284^{* * * *}$ \\
& Oz & -1.48 & $287^{* * * *}$ \\
Right & POz & -1.52 & $287^{* *}$ \\
& PO4 & -1.68 & $290^{* * *}$ \\
& PO6 & -1.45 & $293^{*}$ \\
& PO8 & -1.47 & $293^{*}$ \\
& P4 & -1.49 & $294^{* * *}$ \\
& P2 & -1.29 & $297^{* *}$ \\
\hline \hline
\end{tabular}

$* p<.01 ; * * p<.005 ; * * * p<.0025$.

conditions requiring perception of faces, $\mathrm{P} 1$ being right-located in all conditions but FN and N1 being left-located for the first faces of FF and FN conditions. We cannot completely explain these lateralization effects. Although P1 is generally found to have a bilateral origin (Allison, Puce, Spencer, \& McCarthy, 1999; Rossion et al., 1999; Schweinberger, Pickering, Jentzsch, Burton, \& Jürgen, 2002), interhemispheric differences have also been described in the literature (Small, 1988). On the other hand, it has been suggested in the literature that $\mathrm{P} 1$ and $\mathrm{N} 1$ are two ERP components reflecting the same neural activity (Campanella et al., 2002). Thus, it could suggest that the interaction observed between lateralization and conditions for the P1 and the absence of such interaction for the $\mathrm{N} 1$ do not really reflect functional differences in the cerebral activities involved in the perception of faces between FF and FN conditions.

Whatever, the absence of amplitude or latency differences between mixed and nonmixed conditions for ERP components elicited by the encoding processes of faces and proper names leads us to conclude that all conditions were matched for difficulty and attention. This interpretation is strengthened by the analysis of behavioral data. First, as expected, subjects answered faster and more accurately when the presented pairs of stimuli corresponded to a previously learned couple. Second, latencies of correct responses were identical between mixed and nonmixed conditions. But all percentages of correct responses remained above $92 \%$, probably reflecting a ceiling effect, and, moreover, only correct trials (whose performances were identical

Table 5. Positivity in $(N F+F N)-(N N+F F)$; (1) Hemispheres, (2) Electrodes, (3) Peak Amplitudes, (4) Latency of Maximum Amplitude Difference from Zero (Student $t$ Tests) and Significance Level

\begin{tabular}{llcc}
\hline \hline Electrodes & & Amplitude $(\mu \mathrm{V})$ & Latency $(\mathrm{ms})$ \\
\hline Left & $\mathrm{C} 1$ & 1.06 & $303^{* * * * * *}$ \\
& $\mathrm{FC} 1$ & 1.32 & $300^{* * * * *}$ \\
Midline & $\mathrm{Cz}$ & 1.01 & $310^{* * * *}$ \\
& $\mathrm{FCz}$ & 1.48 & $300^{* * * *}$ \\
Right & $\mathrm{C} 2$ & 0.56 & $302^{* *}$ \\
& $\mathrm{FC} 2$ & 1.09 & $297 * *$ \\
\hline \hline
\end{tabular}

$* p<.1 ; * * p<.05 ; * * * p<.01 ; * * * * * p<.005$.
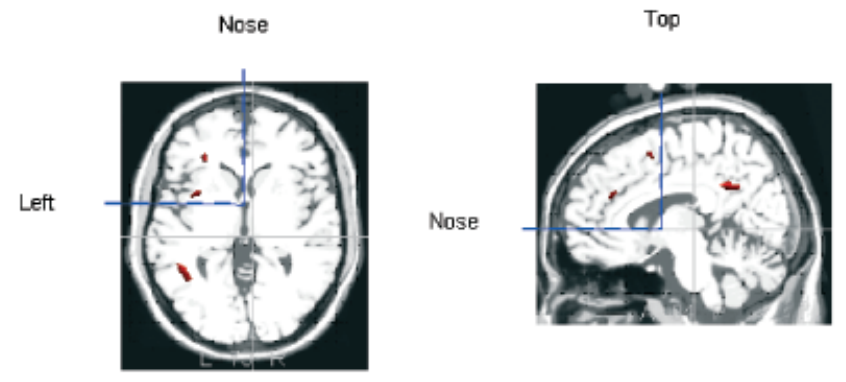

Figure 4. Illustrations of the dipole modelization applied on the grand average of the subtraction $(\mathrm{NF}+\mathrm{FN})-(\mathrm{NN}+\mathrm{FF})$. Length of the dipoles represents their strength. Dipole position (on the down part of the figure, from left to right): IFG 45 ( $x: 36, y: 28, z: 6)$, MFG $6(x: 8, y: 34, z$ : $56)$, and IPL $40(x:-50, y: 44, z: 32)$. Note the most prominent importance of the parietal dipole relative to the two others.

between mixed and nonmixed conditions) were used in our main subtraction. Finally, mean percentages of correct responses and mean correct response latencies were very close to the values observed in the PET study of Campanella et al. (2001). Thus, it seems that the main result of the subtraction cannot be explained by an additional requirement of attentional ressources in mixed conditions in comparison with nonmixed conditions.

The main results of this study derive from the subtraction of FF and NN conditions from FN and NF conditions. First, this analysis was performed to put in light the precise timing of the electrophysiological mechanisms implied in the integrative processes of representations of faces and names, necessary to provide a unified representation of people. Second, on the basis of the PET study of Campanella et al. (2001), we also hypothesized that these neurophysiological activities would be generated in the left hemisphere, and that a dipole localization analysis of the specific integrative waves revealed by the precited subtraction could give prominence to neural generators spatially close from the activated neural regions found by this PET study. The subtraction revealed two main ERP components: (1) a negative wave spreading bilaterally over all posterior electrodes between 280 and $300 \mathrm{~ms}$, but appearing more precociously and with higher statistical significance levels in the left hemisphere (maximum peak at left parietal electrode P5 at $284 \mathrm{~ms}$ ); and (2) its positive counterpart maximally peaking at left centro-frontal sites $(\mathrm{FC} 1 / \mathrm{C} 1)$ around $300 \mathrm{~ms}$. Statistical analysis performed on the negativity showed that even if there were no difference in the wave amplitude between left and right hemispheres, there was a significant latency effect between the two hemispheres, the negativity appearing significantly earlier in the left hemisphere. Thus, we can consider that the main result of this study is that we have showed that processes involved in the integration of visual name and face representations took place in the brain around 280 and $300 \mathrm{~ms}$, with a preferential involvement of the left hemisphere.

Moreover, a dipole modeling showed that $90 \%$ of the variance of these results can be explained by three dipoles corresponding to the neural generators proposed by Campanella et al. (2001). They showed, using the PET technique, the involvement of left parietal (BA 40) and frontal (BA 6 and BA 45 ) regions in the association of different representations of faces and names when subjects had to retrieve a face on the basis of a related name and conversely (Campanella et al., 2001). BA 40 was interpreted as the brain region where the association of the distinct representations presented to subjects operates. The 
activation of the frontal regions was seen as reflecting executive and nonspatial attentional processes (BA 6) and retrieval of the learned associations stored in semantic memory (BA 45).

The present findings seem also to temporally constrain this anatomical model. Indeed, it was possible by considering each of these three dipoles individually to define their precise time of maximal activity. In sight of the results of this dipole modeling, we could propose that (1) the left medial frontal activity, appearing first in time (around $270 \mathrm{~ms}$ ), could reflect the attentional processes necessary to shift from one visual processing (e.g., the processing of a face) to another (the processing of a name; Nagahama et al., 1999); (2) when the perceptual processing of the second stimulus is achieved, its representation could then be integrated to the representation of the first stimulus in the left inferior parietal lobe (around $285 \mathrm{~ms}$ ), seen as a multimodal sensory convergence zone, relevant to perceptual binding and memory retrieval by providing a unique representation of combined sensory features (Clark et al., 2000; Damasio, 1989); finally, (3) this activity could spread, around $300 \mathrm{~ms}$, toward left inferior frontal regions, known to be involved in complex reflexive memory processes (Nolde, Johnson, \& Raye, 1998; Paller, Gonsalves, Grabowecky, Bozic, \& Yamada, 2000; Rugg, Fletcher, Chua, \& Dolan, 1999) such as the selection of information among competing alternatives in semantic memory (Thompson-Schill, D'Esposito, Aguirre, \& Farah, 1997). The comparison of the presented face-name association to one of the couples stored in long-term memory could allow subjects to make a correct decision. As a matter of fact, we have thus a model of face-name retrieval implying sequentially the left frontal medial gyrus, the left inferior parietal lobe, and the left inferior frontal gyrus.

We are aware that the relative limitation of anatomical resolution of the ERP technique and the relative importance of the residual variance for each of our three dipoles in the separate fit analysis do not allow us to claim categorical and definitive conclusions. Moreover, matching $\mathrm{rCBF}$ activations and ERPs waves is a real challenge. On the one hand, $\mathrm{rCBF}$ activations result from the averaging of a multiple cognitive process taking place over an entire block of trials. On the other hand, ERP waves reflect cerebral activities averaged in the time range of single trials. But, overall, it is important to note that the two experiments (the previous PET study and the present ERP one), using different techniques and different participants, lead to very similar results, that is, the existence of a network of cortical areas lateralized in the left hemisphere and producing cerebral activities spreading from posterior to anterior sites when subjects have to retrieve the representation of a face when presented with an associated name and conversely.

If the neuroanatomical and the neurophysiological correlates of face perception begin to be well known, our experiments are, however, among the rare studies that have investigated the integration and retrieval of face-name associations. Our results raise many interesting questions and further research will be necessary to provide a better comprehension of these processes, notably about the implication of our neural network in cross-modal integration processing (such as visuo-auditive face-name integration) or the specificity of this network relative to more general binding processes (such as object-common name associations).

\section{REFERENCES}

Allison, T., Puce, A., Spencer, D. D., \& McCarthy, G. (1999). Electrophysiological studies of human face perception I. Potentials generated in occipitotemporal cortex by face and non-face stimuli. Cerebral Cortex, 9, 415-430.

Bentin, S., Allison, T., Puce, A., Perez, E., \& McCarthy, G. (1996). Electrophysiological studies of face perception in humans. Journal of Cognitive Neuroscience, $8,551-565$.

Bentin, S., Mouchetant-Rostaing, Y., Giard, M. H., Echallier, J. F., \& Pernier, J. (1999). ERP manifestations of processing printed words at different psycholinguistic levels: Time course and scalp distribution. Journal of Cognitive Neuroscience, 11, 235-260.

Bertrand, O., Perrin, F., \& Perrier, J. (1985). A theorical justification of the average reference in topographic evoked potentials studies. Clinical Neurophysiology, 62, 462-464.

Bodamer, J. (1947). Die Prosop-Agnosia (Die Agnosie des Physionomeerkennens). Archiv für Psychiatrie und Nervenkrankenheiten, 179, 6-33.

Bötzel, K., Schulze, S., \& Stodieck, R. G. (1995). Scalp topography and analysis of intracranial sources of face-evoked potentials. Experimental Brain Research, 104, 135-143.

Campanella, S., Hanoteau, C., Depy, D., Rossion, B., Bruyer, R., Crommelinck, M., \& Guérit, J. M. (2000). Right N170 modulation in a face discrimination task: An account for categorical perception of familiar faces. Psychophysiology, 37, 796-806.

Campanella, S., Joassin, F., Rossion, B., De Volder, A. G., Bruyer, R., \& Crommelinck, M. (2001). Association of the distinct visual representations of faces and names: A PET activation study. NeuroImage, $14,873-882$.

Campanella, S., Quinet, P., Bruyer, R., Crommelinck, M., \& Guérit, J. M. (2002). Categorical perception of happiness and fear facial expressions: An ERP study. Journal of Cognitive Neuroscience, 14(2), 1-18.

Clark, C. R., Egan, G. F., McFarlane, A. C., Morris, P., Weber, D., Sonkkilla, C., Marcina, J., \& Tochon-Danguy, H. J. (2000). Updating working memory for words: A PET activation study. Human Brain Mapping, 9(1), 42-54.
Cohen, N. J., Ryan, J., Hunt, C., Romine, L., Wszalek, T., \& Nash, C. (1999). Hippocampal system and declarative (relational) memory: Summarizing the data from functional neuroimaging studies. Hippocampus, 9, 83-98.

Coull, J. T. (1998). Neural correlates of attention and arousal: Insights from electrophysiology, functional neuroimaging and psychopharmacology. Progress in Neurobiology, 55, 343-361.

Damasio, A. R. (1989). Time-locked multiregional retroactivation: A system-level proposal for neural substrates of recall and recognition. Cognition, 33, 25-62.

Damasio, A. R., Damasio, H., Tranel, D., \& Brandt, J. P. (1990). The neural regionalization of knowledge access: Preliminary evidence. In Cold Spring Harbor Symposia on Quantitative Biology, Vol. 60: The Brain (pp. 1039-1047). Cold Spring Harbor, NY: Cold Spring Harbor Laboratory Press.

Damasio, H., Grabowski, T. J., Tranel, D., Hichwa, R. D., \& Damasio, A. R. (1996). A neural basis for lexical retrieval. Nature, 380, 499-505.

Dehaene, S. (1995). Electrophysiological evidence for category-specific word processing in the normal human brain. NeuroReport, 6, 2153-2157.

Dehaene, S., Naccache, L., Cohen, L., Le Bihan, D., Mangin, J. F., Poline, J. B., \& Rivière, D. (2001). Cerebral mechanisms of word masking and unconscious repetition priming. Nature Neuroscience, 4, 752-758.

De Renzi, E., Peroni, D., Carlesimo, G. A., Silveri, M. C., \& Fazio, F. (1994). Prosopagnosia can be dissociated with damage confined to the right hemisphere-An MRI and PET study and a review of the literature. Neuropsychologia, 32, 893-902.

Dubois, S., Rossion, B., Schiltz, C., Bodart, J. M., Michel, C., Bruyer, R., \& Crommelinck, M. (1999). Effect of familiarity on the processing of human faces. NeuroImage, 9, 278-289.

George, N., Evans, J., Fiori, N., Davidoff, J., \& Renault, B. (1996). Brain events related to normal and moderately scrambled faces. Cognitive Brain Research, 4, 65-76.

Giard, M. H., \& Peronnet, F. (1999). Auditory-visual integration during multimodal object recognition in humans: A behavioral and 
electrophysiological study. Journal of Cognitive Neuroscience, 11, 473-490.

Gomez, C. M., Clark, V. P., Luck, S. J., Fan, S., \& Hillyard, S. A. (1994). Sources of attention sensitive visual event-related potentials. Brain Topography, 7, 41-51.

Haxby, J. V., Horwitz, B., Ungerleider, L. G., Maisog, J. M., Rapoport, S. I., \& Grady, C. L. (1994). The functional organization of human extrastriate cortex: A PET-rCBF study of selective attention to faces and locations. Journal of Neuroscience, 14, 6336-6353.

Heinze, H. J., Mangun, G. R., Burchert, W., Hinrichs, H., Scholtz, M., Münte, T. F., Gös, A., Scherg, M., Johannes, S., Hundeshagen, H., Gazzaniga, M. S., \& Hillyard, S. A. (1994). Combined spatial and temporal imaging of brain activity during selective attention in humans. Nature, 372, 543-546.

Itier, R. J., \& Taylor, M. J. (2002). Inversion and contrast polarity reversal affect both encoding and recognition processes of unfamiliar faces: A repetition study using ERPs. NeuroImage, 15, 353-372.

Jeffreys, D. A. (1989). A face-responsive potential recorded from the human scalp. Experimental Brain Research, 78, 193-202.

Jeffreys, D. A. (1996). Evoked potentials studies of face and object processing. Visual Cognition, 3, 1-38.

Kanwisher, N., McDermott, J., \& Chun, M. M. (1997). The fusiform face area: A module in human extrastriate cortex specialized for face perception. Journal of Neuroscience, 9, 462-475.

Kim, J. J., Andreasen, N. C., O’Leary, D. S., Wiser, A. K., Boles Ponto, L. L., Watkins, G. L., \& Hichwa, R. D. (1999). Direct comparison of the neural substrates of recognition memory for words and faces. Brain, 122, 1069-1083.

Leveroni, C. L., Seindenberg, M., Mayer, A. R., Mead, L. A., Binder, J. R., \& Rao, S. M. (2000). Neural systems underlying the recognition of familiar and newly learned faces. Journal of Neuroscience, 20, 878-886.

Müller, H., \& Kutas, M. (1996). What's in a name? Electrophysiological differences between spoken nouns, proper names and one's own name. NeuroReport, 8, 221-225.

Nagahama, Y., Okada, T., Katsumi, Y., Hayashi, T., Yamauchi, H., Sawamoto, N., Toma, K., Nakamura, K., Hanakawa, T., Konishi, J., Fukuyama, H., \& Shibasaki, H. (1999). Transient neural activity in the medial superior frontal gyrus and precuneus time-locked with attention shift between object features. NeuroImage, 10, 193-199.

Nolde, S. F., Johnson, M. K., \& Raye, C. L. (1998). The role of the prefrontal cortex during tests of episodic memory. Trends in Cognitive Science, 2, 399-406.

Paller, K. A., Gonsalves, B., Grabowecky, M., Bozic, V. S., \& Yamada, S. (2000). Electrophysiological correlates of recollecting faces of known and unknown individuals. NeuroImage, 11, 98-110.

Papagno, C., \& Capitani, E. (1998). Proper name anomia: A case with sparing of the first-letter knowledge. Neuropsychologia, 36, 669-679.

Price, J. C., Moore, C. J., Humphreys, G. W., Frackowiak, R. S. J., \& Friston, K. J. (1996). The neural pathways sustaining object and colour naming. Proceedings of the Royal Society of London B, Biological Science, 263, 1501-1507.

Price, J. C., Moore, C. J., Humphreys, G. W., \& Wise, R. J. (1997). Segregating semantic from phonological procesess during reading. Journal of Cognitive Neuroscience, 9, 727-733.
Proverbio, A. M., Lilli, S., Semenza, C., \& Zani, A. (2001). ERP indexes of functional differences in brain activation during proper and common names retrieval. Neuropsychologia, 39, 815-827.

Puce, A., Allison, T., Asgari, M., Gore, J. C., \& McCarthy, G. (1996). Differential sensitivity of human visual cortex to faces, letter-strings and textures: A functional magnetic resonance imaging study. Journal of Neuroscience, 16, 5205-5215.

Puce, A., Allison, T., Gore, J. C., \& McCarthy, G. (1995). Face-sensitive regions in human extrastriate cortex studied by functional MRI. Journal of Neurophysiology, 74, 1192-1199.

Reinkemeier, M., Markowitsch, H. J., Rauch, M., \& Kessler, J. (1997). Differential impairments in recalling people's names: A case study in search of neuroanatomical correlates. Neuropsychologia, 35, 677-684.

Rossion, B., Campanella, S., Guérit, J. M., Debatisse, D., Liard, L., Gomez, C., Dubois, S., Delinte, A., Bruyer, R., \& Crommelinck, M. (1999). Task modulation of brain activity related to familiar and unfamiliar face processing: An ERP study. Clinical Neurophysiology, 110, 449-462.

Rugg, M. D., \& Coles, M. (1995). Electrophysiology of mind. Oxford: Oxford University Press.

Rugg, M. D., Doyle, M. C., \& Wells, T. (1995). Word and non-word repetition within and across modality: An event-related potential study. Journal of Cognitive Neuroscience, 7, 209-227.

Rugg, M. D., Fletcher, P. C., Chua, P. M., \& Dolan, R. J. (1999). The role of the prefrontal cortex in recognition memory and memory for sources: An fMRI study. NeuroImage, 10, 520-529.

Schweinberger, S. R., Pickering, E. C., Jentzsch, I., Burton, A. M., \& Jürgen, M. K. (2002). Event-related potential evidence for a response of inferior temporal cortex to familiar face repetitions. Brain Research: Cognitive Brain Research, 14, 398-409.

Semenza, C., \& Zettin, M. (1988). Generating proper names: A case of selective inability. Cognitive Neuropsychology, 5, 711-721.

Sergent, J., Ohta, S., \& McDonald, B. (1992). Functional neuroanatomy of face and object processing. Brain, 115, 15-36.

Small, M. (1988). Visual evoked potentials in a patient with prosopagnosia. Electroencephalography and Clinical Neurophysiology, 71, $10-16$.

Sperling, R. A., Bates, J. F., Cocchiarella, A. J., Schachter, D. L., Rosen, B. R., \& Albert, M. S. (2001). Encoding novel face-name associations: A functional MRI study. Human Brain Mapping, 14, 129-139.

Takahashi, N., Kawamura, M., Hirayama, K., Shiota, J., \& Tsono, O. (1995). Prosopagnosia: A clinical and anatomical study of four patients. Cortex, 31, 317-329.

Tempini, M. L. G., Price, C. J., Josephs, O., Vandenberghe, R., Cappa, S. F., Kappur, N., \& Frackowiak, R. S. J (1998). The neural systems sustaining face and proper name processing. Brain, 121, 2103-2118.

Thompson-Schill, S. L., D'Esposito, M., Aguirre, G. K., \& Farah, M. J. (1997). Role of left inferior frontal cortex in retrieval of semantic knowledge: A reevaluation. Proceedings of the National, 94, 14792-14797.

(Received November 26, 2002; ACCEPTED October 29, 2003) 